\title{
Transduction Analysis of the Histidine Region in Staphylococcus aureus
}

\author{
By W. E. KLOOS and P. A. PATTEE \\ Department of Bacteriology, Iowa State University, Ames, Iowa, U.S.A.
}

(Received 21 October 1964)

\begin{abstract}
SUMMARY
The genetic control of histidine biosynthesis in Staphylococcus aureus was examined by an analysis of 66 histidine-dependent mutants by using stable and abortive transduction. These mutants, which previously had been differentiated into classes according to their biochemical characteristics, all occupied sites within a single linkage group, referred to as the histidine region. This region has been separated into six gene loci on the basis of complementation studies and the biochemical characteristics of the mutants. The order of genes within the histidine region, $E, A, B, C$, $D, G$, was determined from the results of reciprocal transductions to wild type and donor type. The order of mutant sites was also linearly related to the ability of these mutants to form histidine-independent transductants when infected with phage prepared on the parent strain. This observation, and the inequalities in reciprocal transduction frequencies obtained in inter-mutant transductions, support the hypothesis that all donor fragments which participate in transductions involving the histidine region are identical, and that the histidine region is located extremely close to one terminus of the donor fragment. Intergenic complementation occurred among mutants of all gene loci; in addition, three complementation units within the $A$ gene and two complementation units within the $G$ gene were detected.
\end{abstract}

\section{INTRODUCTION}

A genetic analysis of seven histidine-dependent mutants of Neurospora crassa revealed the existence of three linkage groups and four different gene loci (Haas, Mitchell, Ames \& Mitchell, 1952). Webber \& Case (1960) extended this analysis to include over 700 mutants of $N$. crassa. In these studies, representative mutants from each of seven biochemical groups were analysed for their linkage relationships and were found to represent seven different histidine loci which formed five linkage groups. In contrast to the wide distribution of histidine loci throughout the genome of $N$. crassa, a cluster of four adjacent histidine loci was found in Salmonella typhimurium (Hartman, 1956). Additional investigations were conducted which involved about 1000 histidine-dependent mutants (Hartman, Loper \& Serman, 1960; Ames \& Hartman, 1962, 1963). By means of transduction, a genetic map of the histidine operon was constructed which consisted of eight adjacent loci in the sequence $E, F, A, H, B, C, D, G$. By abortive transduction, Hartman, Hartman \& Serman (1960) determined the functional relationships of over 200 histidine-dependent mutants. The results of this analysis divided the mutants into eight classes, $A$ to $H$. In addition, intragenic complementation was found 
to occur among the $E, B$ and $D$ mutants. The $E$ and $B$ genes were composed of four complementation units each $(a, b, c, d)$. The $D$ gene was composed of two complementation units $(a, b)$. Loper $(1960,1961)$ analysed the $B$ gene in more detail and found that 23 mutants of the $B c$ complementation unit lacked both the imidazoleglycerol phosphate dehydrase and the histidinol phosphate phosphatase enzymes, whereas a large number of $B a, B b$ and $B d$ mutants lacked only the imidazoleglycerol phosphate dehydrase. More recently, Smith \& Ames (1964) showed that $E a$ and $E d$ mutants could be differentiated from the $E b$ and $E c$ mutants. The former mutants now are designated $E$ mutants, while the $E b$ and $E c$ mutants now are referred to as $I$ mutants.

Ephrati-Elizur, Srinivasan \& Zamenhof (1961) studied the histidine region of the chromosome of Bacillus subtilis by means of transformation. These investigators determined that twelve histidine-dependent mutants represented two loci, $A$ and $D$. Because mutants of the $A$ locus were not closely linked to the $D$ locus, it was suggested that the region between these two loci might be occupied by genes which controlled other steps in the biosynthesis of L-histidine.

The present paper describes a transduction analysis of histidine-dependent mutants of Staphylococcus aureus. A unique method of mapping the histidine loci of $S$. aureus is described which is based upon the frequency with which the mutant strains are transduced to histidine independence when the parent strain is used as the donor.

\section{METHODS}

Media. The media used in this study included Brain Heart Infusion (BHI; Difco) agar, $\mathbf{P}$ and $\mathbf{D}$ broth (Pattee \& Baldwin, 1961), S agar (Kloos \& Pattee, 1965) and an enriched defined medium agar (ES agar) prepared by the addition of $1.0 \%(\mathrm{v} / \mathrm{v}) \mathrm{P}$ and $\mathrm{D}$ broth to $\mathrm{S}$ agar. All glassware used in conjunction with the defined media was cleaned in chromic + sulphuric acid and thoroughly rinsed with deionized water before use.

Bacterial strains. This study was done exclusively with strain 655 of Staphylococcus aureus (Pattee \& Baldwin, 1962) and with histidine-dependent mutants derived either from this strain or from a single penicillinase-deficient mutant of this strain. These mutants, the nomenclature used in their designation, and their biochemical characteristics are described by Kloos \& Pattee (1965).

Transduction. In all instances bacteriologically-sterile lysates of phage 83 previously propagated on Staphylococcus aureus strain 655 (designated phage 83/655) or on appropriate mutants of strain 655 were used for transduction. The methods used to prepare these lysates were described by Pattee \& Baldwin (1961). These lysates were prepared from phage 83 which had been maintained on the appropriate International strain of Staphylococcus aureus. Serial passage of phage 83 on $S$. aureus strain 655 was avoided as this practice resulted in a loss of transductional activity.

Cultures to be used as recipients in transduction experiments were grown on BHI agar slopes for $18 \mathrm{hr}$ at $37^{\circ}$. Each culture was suspended in $1.0 \mathrm{ml}$. of $\mathrm{P}$ and $\mathrm{D}$ broth (coccal concentration $=5-7 \times 10^{10} \mathrm{cocci} / \mathrm{ml}$.) and $0.5 \mathrm{ml}$. of this suspension transferred to a centrifuge tube containing $1.0 \mathrm{ml}$. $P$ and $D$ broth. To this suspension was added $0.5 \mathrm{ml}$. of the transducing phage lysate which had been diluted previously with $\mathbf{P}$ and $\mathbf{D}$ broth when necessary, to obtain a multiplicity of infection in the 
transduction suspension of $\mathbf{2} \cdot \mathbf{0}-\mathbf{3} \cdot \mathbf{5}$. Controls consisted of identical coccal suspensions, to which $0.5 \mathrm{ml} . \mathrm{P}$ and $\mathrm{D}$ broth was added in place of the phage lysate. The suspensions were shaken for $30 \mathrm{~min}$. at $37^{\circ}$ by means of a Burrell Wrist-Action Shaker (2223 Fifth Avenue, Pittsburg, Pa., U.S.A.) with the arms mounted over water baths. The maximum shaker setting of 10 was used. After shaking, the cocci were collected by centrifugation and resuspended in $1.0 \mathrm{ml}$. saline. Duplicate $0.1 \mathrm{ml}$. samples from a 1/10 dilution of each suspension were then spread over the surfaces of pre-dried ES agar plates. The plates were incubated at $37^{\circ}$ for $48 \mathrm{hr}$, after which the average numbers of histidine-independent and donor-type transductants per sample were determined. Abortive transductants were detected by spreading $\mathbf{0} \cdot \mathbf{1} \mathrm{ml}$. samples of the undiluted transduction suspension over pre-dried plates of $\mathrm{S}$ agar. After incubation of these plates for $48 \mathrm{hr}$ at $37^{\circ}$, they were examined with a dissecting microscope for abortive transductants, as indicated by the presence of 'minute' colonies (Ozeki, 1959).

In all experiments, the transduction frequency was recorded as the average number of colonies recovered from duplicate $0.1 \mathrm{ml}$. samples of the $1 / 10$ dilution of the transduction suspension. The reversion frequency was recorded as the average number of colonies recovered from duplicate $0 \cdot 1 \mathrm{ml}$. samples of the $1 / 10$ dilution of the control suspension.

\section{RESULTS}

\section{Effect of the multiplicity of infection on the transduction frequency}

Among those bacteriophages of the International Typing Series which could be propagated to high titre on Staphylococcus aureus strain 655 (phages 83, 53, 54, 77, 47 ), phage 83 possessed the highest activity in transducing histidine independence to histidine-dependent mutants of strain 655. Preliminary studies with phage 83 showed that the multiplicity of infection used in the transduction suspension influenced the transduction frequency to a significant extent. With phage 83/655 and three recipient strains ( $h i s A b-1, h i s D-8, h i s G b-15)$, when the multiplicity of infection was maintained between $2 \cdot 0$ and $3 \cdot 5$, maximum and reproducible transduction frequencies were obtained. Accordingly, all experiments were made with transduction suspensions infected at these multiplicities.

\section{Recipient capacity, reversion frequency and incidence of abortive transduction}

All histidine-dependent mutants were infected with phage 83/655 to determine the recipient capacity (efficiency to produce prototrophic transductants) and the reversion frequency of each mutant, and to determine which mutants were capable of undergoing abortive transduction. The experiment was made with a single lysate of phage $83 / 655$ so that the titre of the transducing phage lysate could be eliminated as a factor responsible for variations in the recipient capacities of the mutants. The results of this experiment are shown in Table 1. The mutants listed in the legend of Table 1 were discarded from further use in genetic studies either because of their high reversion frequencies or because of their leakiness, or of both. Those mutants which were not analysed by transduction have been designated solely according to their biochemical characteristics (Kloos \& Pattee, 1965). With the exception of mutant hisAaD-63, none of the $D$ mutants gave rise to detectable abortive 
transductants when coccal suspensions infected with phage $83 / 655$ were inoculated on to either $\mathbf{S}$ agar or ES agar. However, after the $\mathbf{S}$ agar plates inoculated with phage-infected suspensions of $D$ mutants had been incubated for $48 \mathrm{hr}$, the addition of a small amount of crystalline L-histidine (monohydrate; California Corporation

\section{Table 1. Frequency of transduction of histidine independence to histidine-dependent mutants of Staphylococcus aureus by phage 83/655}

Phage 83/655 was used to infect $2 \cdot 5-3 \cdot 5 \times 10^{10}$ cocci of each recipient at a multiplicity of $2 \cdot 0-3 \cdot 5$. The transduction frequency is the average number of prototrophic transductants recovered per $0.1 \mathrm{ml}$. of a $1 / 10$ dilution of the transduction suspension inoculated in duplicate on ES agar. The reversion frequency is the average number of prototrophic colonies recovered from uninfected coccal suspensions which were diluted and plated in the same manner as the transduction suspensions. The capacity of a mutant to undergo abortive transduction was determined on $\mathrm{S}$ agar. The presence of abortive transductants is indicated by a + symbol, and the failure of a mutant to give rise to abortive transductants without the addition of L-histidine is indicated by a - symbol. See text for details of the histidine stimulation procedure. Mutants found to be excessively leaky, or to revert at frequencies excessively high for use in genetic analyses, or both, include mutants his : D-16, D-25, B-27, G-29, G-30, G-34, G-35, G-37, G-39, G-45, C-48, D-51, A-64, $G-65, D-70, A-77, G-78, D-82, G-87, D-88, G-89, C-91, G-94, G-95, C-101$ and $D-103$.

$\begin{array}{cccc} & \begin{array}{c}\text { Trans- } \\ \text { duction } \\ \text { fre- } \\ \text { Mutant }\end{array} & \begin{array}{c}\text { Presence } \\ \text { of } \\ \text { quency } \\ \text { abortive } \\ \text { trans- } \\ \text { frequency } \\ \text { ductants }\end{array}\end{array}$

\begin{tabular}{|c|c|}
\hline his $A b-1$ & 136 \\
\hline hisB-2 & 332 \\
\hline hisD-3 & 474 \\
\hline$h i s A b-5$ & 119 \\
\hline$h i s A b-6$ & 125 \\
\hline his $A b-7$ & 131 \\
\hline hisD-8 & 446 \\
\hline$h i s G b-9$ & 1192 \\
\hline his $A b-10$ & 136 \\
\hline his Aa-11 & 140 \\
\hline $\operatorname{his} A b-12$ & 151 \\
\hline hisGb-13 & 1368 \\
\hline hisC-14 & 401 \\
\hline$h i s G b-15$ & 1300 \\
\hline his $A b-17$ & 145 \\
\hline hisD-18 & 407 \\
\hline his $A b-19$ & 113 \\
\hline his $A b-20$ & 142 \\
\hline hisC-23 & 518 \\
\hline hisD-24 & 419 \\
\hline $\operatorname{his} A a-31$ & 132 \\
\hline hisD-32 & 503 \\
\hline hisD-33 & 499 \\
\hline$h i s D-36$ & 397 \\
\hline hisGb-38 & 1389 \\
\hline hisGa-40 & 1336 \\
\hline hisB-41 & 215 \\
\hline hisD-42 & 677 \\
\hline hisD-43 & 457 \\
\hline hisD-44 & 425 \\
\hline hisD-46 & 513 \\
\hline$h i s D-47$ & 575 \\
\hline hisE-49 & 118 \\
\hline
\end{tabular}

$\begin{array}{rr}\mathbf{2} & + \\ \mathbf{1 4} & + \\ \mathbf{0} & + \\ \mathbf{4} & + \\ \mathbf{2} & + \\ \mathbf{4} & + \\ \mathbf{2} & + \\ \mathbf{1 0} & + \\ \mathbf{1 0} & + \\ \mathbf{1 0} & + \\ \mathbf{1} & + \\ \mathbf{2} & + \\ \mathbf{3} & + \\ \mathbf{2} & + \\ \mathbf{2} & + \\ \mathbf{3} & + \\ \mathbf{1} & + \\ \mathbf{2} & + \\ \mathbf{1 5} & + \\ \mathbf{1} & + \\ \mathbf{3} & + \\ \mathbf{0} & + \\ \mathbf{1} & + \\ \mathbf{1} & + \\ \mathbf{1 7} & + \\ \mathbf{2 3} & + \\ \mathbf{2} & + \\ \mathbf{4} & + \\ \mathbf{1} & + \\ \mathbf{0} & + \\ \mathbf{2} & + \\ \mathbf{7} & +\end{array}$

+
+
-
+
+
+
+
+
+
+
+
+
+
+
+
-
+
+
+
+
+
-
-
-
+
+
+
-
-
-
+
+

Mutant

hisC-50
hisD-52
hisD-54
hisD-55
hisC-56
hisD-58
hisD-59
hisD-60
hisD-61
hisGa-62
hisAaD-6
hisC-66
hisD-68
hisD-69
hisD-71
hisD-71
hisC-73
hisGa-74
hisGa-75
hisAc-76
hisAa-79
his $A-80$
hisD-81
hisAa-83
hisD-84
hisD-85
hisC-90
hisE-93
hisC-96
hisD-98
hisGb-99
hisD-100
hisD-104

hisD-104

$\begin{array}{cc}\text { Trans- } & \\ \text { duction } & \\ \text { fre- } & \text { Reversion } \\ \text { quency } & \text { frequency }\end{array}$

Presence of abortive transductants

$\begin{array}{rrr}\mathbf{3 6 8} & \mathbf{2} & + \\ \mathbf{5 0 9} & \mathbf{2} & - \\ \mathbf{5 2 9} & \mathbf{5} & - \\ \mathbf{5 3 8} & 1 & - \\ \mathbf{3 7 0} & \mathbf{1 2} & + \\ \mathbf{3 3 4} & \mathbf{0} & - \\ \mathbf{5 5 2} & \mathbf{4} & - \\ \mathbf{4 9 2} & \mathbf{5} & - \\ \mathbf{3 4 3} & \mathbf{0} & - \\ \mathbf{1 3 3 9} & \mathbf{1 8} & + \\ \mathbf{6 7} & \mathbf{0} & + \\ \mathbf{4 8 2} & \mathbf{5 5} & + \\ \mathbf{5 9 0} & \mathbf{0} & - \\ \mathbf{6 1 6} & \mathbf{0} & - \\ \mathbf{4 8 8} & \mathbf{0} & - \\ \mathbf{5 4 1} & \mathbf{3} & - \\ \mathbf{3 2 2} & \mathbf{7} & + \\ \mathbf{9 2 4} & \mathbf{1 0} & + \\ \mathbf{1 1 7 0} & \mathbf{3} & + \\ \mathbf{1 4 1} & \mathbf{0} & + \\ \mathbf{1 5 2} & \mathbf{3} & + \\ \mathbf{1 8 2} & \mathbf{6 0} & + \\ \mathbf{3 5 9} & \mathbf{0} & - \\ \mathbf{1 2 7} & \mathbf{2} & + \\ \mathbf{5 0 6} & \mathbf{0} & - \\ \mathbf{6 2 7} & \mathbf{8} & - \\ \mathbf{3 2 8} & \mathbf{1} & + \\ \mathbf{1 0 0} & \mathbf{2 7} & + \\ \mathbf{3 5 8} & \mathbf{2 0} & + \\ \mathbf{3 4 7} & \mathbf{2} & - \\ \mathbf{9 9 0} & \mathbf{2 3} & + \\ \mathbf{3 6 1} & \mathbf{0} & - \\ \mathbf{4 3 4} & \mathbf{0} & - \\ & & \end{array}$




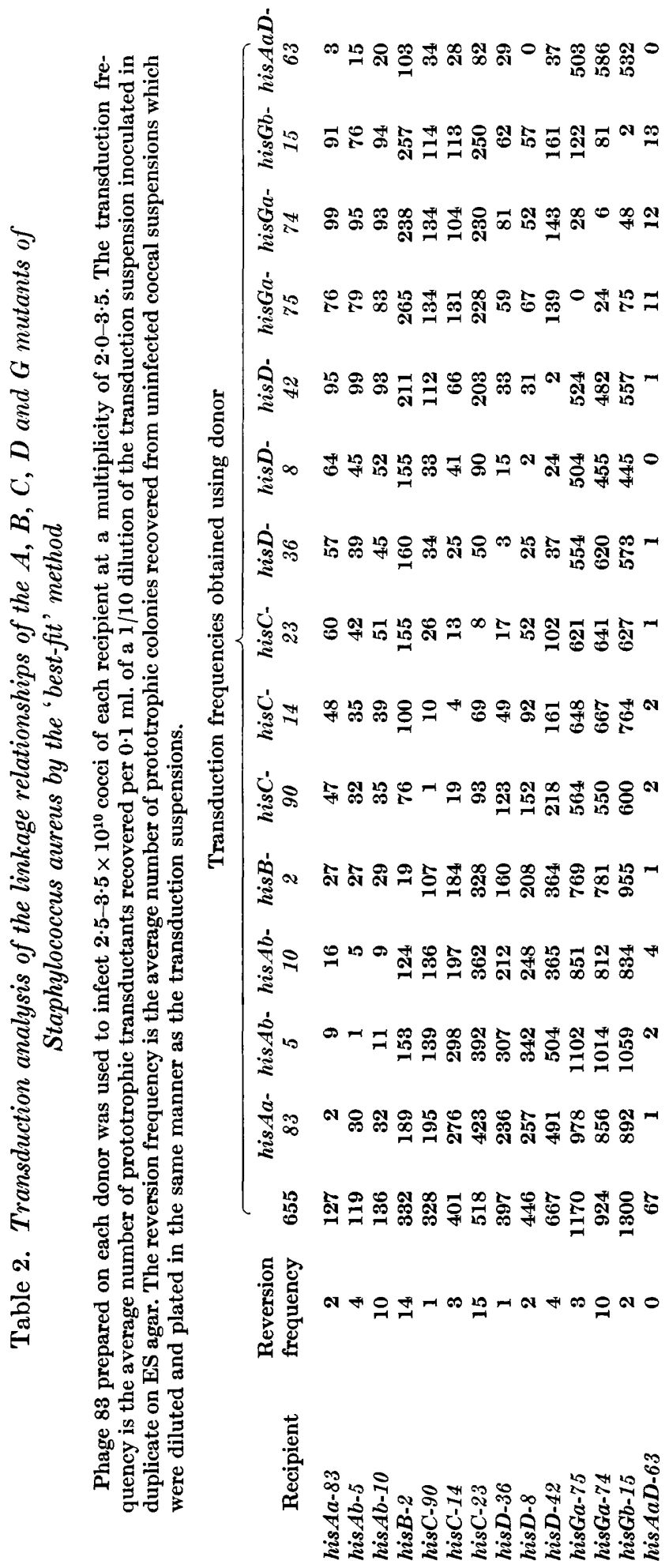


for Biochemical Research, Los Angeles, Calif.) to the agar surface permitted detectable growth of the abortive transductants.

\section{Determination of the order of mutant sites within the histidine region by the 'best-fit' method}

The order of mutant sites within the histidine region was determined by the 'bestfit' method (Hartman, Loper \& Serman, 1960). In this method, the frequencies with which all possible pairs of mutants underwent recombination, as determined by transduction, were used to define a single order of mutant sites which best agreed with all the data. In the present study, three mutants from each class were used in the 'best-fit' method of analysis, with the exceptions of class $E$ which was omitted, and class $B$ for which only one mutant was suitable for use. These mutants were chosen for their low reversion frequencies and absence of leakiness, although mutants his Aa-83 and hisC-90 were slightly leaky; mutant his AaD-63 also was included in the experiment. Lysates of phage 83 prepared on each mutant and on Staphylococcus aureus strain 655 were used to infect each mutant, and the numbers of transductants obtained on ES agar were determined. The results of this experiment are shown in Table 2. The order of mutants as they are shown in Table 2, with the exception of mutant his $A a D-63$, represents the order of mutant sites as determined from the results of this experiment. The histidine-dependent mutants exhibited considerable variation in recipient capacity, as is evident from the transduction frequencies obtained with phage 83/655. For this reason, an analysis of the transduction data after correcting it for variations in recipient capacity was not made. Instead, all the transduction frequencies obtained with a single recipient were analysed for the most probable order of mutant sites, and this analysis repeated for each recipient. These results were then compiled to determine the order of mutant sites which best satisfied all the data. The results indicate that those mutants which exhibited similar accumulation patterns (Kloos \& Pattee, 1965) were also closely linked to one another. From a consideration of the results obtained with mutant his AaD-63, this mutant appears to be a double mutant. This conclusion is supported by the observation that this mutant accumulated 5-amino1-ribosyl-4-imidazolecarboxamide, but did not utilize exogenous L-histidinol (Kloos \& Pattee, 1965) and by the results of complementation studies which are presented below.

\section{Determination of the position of mutant sites relative to the $D$ locus by the ratio test}

To obtain further information concerning the arrangement of mutant sites within the histidine region, all single-site mutants used previously in the 'best-fit' analysis, as well as mutant hisE-49, were analysed by the ratio test (Hartman, Loper \& Serman, 1960). In the ratio test, the numbers of donor-type and wild-type transductants obtained after infection of one mutant with phage grown on another mutant were determined simultaneously from samples of the transduction suspension spread on ES agar. The results are expressed in terms of the probability $(p)$ of integration, by the recipient, of the wild-type allele of the donor without the simultaneous integration of the mutant allele of the donor. Therefore, $p$ is equal to the numbers of wild-type transductants divided by the sum of the wild- 
type and donor-type transductants. Mutants hisD-36, hisD-8 and hisD-42 were chosen for use as recipients since they secrete sufficient histidinol into the environment to support the growth of all other mutant classes. After infection of the $D$ mutants with lysates of phage 83 prepared on other mutants, samples of the transduction suspension were spread on ES agar in the usual manner. Wild-type transductants were differentiated from donor-type transductants by their larger size and more intense pigmentation. The results of this experiment (Table 3) agree with the order of mutant sites as determined by the 'best-fit' method of analysis. Moreover, the results provide conclusive evidence for the existence of the entire histidine region as a single linkage group which is carried on a single donor fragment during transduction. Evidence that the smaller weakly-pigmented colonies were donor-type transductants includes the following facts: (1) no donor-type transductants were obtained when any $D$ mutant was infected with phage prepared on any other $D$ mutant; (2) the donor-type transductants were stimulated by L-histidinol (dihydrochloride; Mann Research Laboratories, New York, N.Y.); (3) the donortype transductants did not undergo recombination with the strain from which their mutant allele was acquired, and yet did produce wild-type recombinants at predictable frequencies when they were transduced with phage 83 prepared on other mutants.

Table 3. The probability $(p)$ of independent integration of different histidine markers into class $\mathbf{D}$ mutant recipient strains of Staphylococcus aureus

Phage 83 prepared on each donor was used to infect $2.5-3.5 \times 10^{10}$ cocci of each recipient at a multiplicity of $2 \cdot 0-3 \cdot 5$. The numbers of wild-type and donor-type transductants recovered from $0.1 \mathrm{ml}$. samples of $1 / 10$ dilutions of the transduction suspension spread on ES agar were determined from duplicate platings. The numbers (no.) columns indicate the total number of transductants scored to determine the value of $p$ in each experiment. ( $p=$ numbers of wild-type transductants divided by the numbers of wild-type + donor-type transductants.)

\begin{tabular}{|c|c|c|c|c|c|c|}
\hline \multirow[b]{3}{*}{ Donor } & \multicolumn{6}{|c|}{ Recipients used } \\
\hline & \multicolumn{2}{|c|}{$h i s D-36$} & \multicolumn{2}{|c|}{ hisD- 8} & \multicolumn{2}{|c|}{ hisD-42 } \\
\hline & $p$ & No. & $p$ & No. & $p$ & No. \\
\hline hisE-49 & $0 \cdot 86$ & 289 & $0 \cdot 85$ & 266 & 0.93 & 532 \\
\hline $\operatorname{his} A a-83$ & $0 \cdot 84$ & 267 & $0 \cdot 85$ & 300 & 0.88 & 554 \\
\hline$h i s A b-5$ & $0 \cdot 77$ & 399 & $0 \cdot 80$ & 426 & $0 \cdot 85$ & 589 \\
\hline$h i s A b-10$ & $0 \cdot 79$ & 267 & $0 \cdot 77$ & 322 & $0 \cdot 83$ & 421 \\
\hline hisB-2 & 0.56 & 290 & $0 \cdot 60$ & 347 & $0 \cdot 63$ & 737 \\
\hline hisC-90 & 0.50 & 246 & $0 \cdot 45$ & $\mathbf{3 3 3}$ & 0.53 & 377 \\
\hline hisC-14 & $0 \cdot 12$ & 406 & $0 \cdot 22$ & 427 & $0 \cdot 27$ & 594 \\
\hline hisC-23 & $0 \cdot 05$ & 350 & $0 \cdot 11$ & 452 & $0 \cdot 16$ & 651 \\
\hline hisGa-75 & $0 \cdot 17$ & 268 & $0 \cdot 19$ & 332 & $0 \cdot 21$ & 673 \\
\hline hisGa-74 & 0.21 & 462 & $0 \cdot 20$ & 508 & 0.19 . & 711 \\
\hline hisGb-15 & $0 \cdot 27$ & 259 & $0 \cdot 20$ & 256 & 0.23 & 695 \\
\hline
\end{tabular}

\section{Complementation studies}

All the histidine-dependent mutants produced abortive transductants when infected with phage $83 / 655$, although the $D$ mutants required the delayed addition of L-histidine to the $\mathbf{S}$ agar plates to facilitate their development. That the minute colonies were the result of abortive transduction is supported by their morphology 
$(0 \cdot 02-0 \cdot 10 \mathrm{~mm}$. diam.), their failure to appear when a mutant was infected with phage 83 previously prepared on that mutant, and their failure to produce more than a single minute colony when subcultured on $\mathrm{S}$ agar plates.

To characterize the functional units of the histidine region, the mutants used in the 'best-fit' analysis were examined for their ability to complement one another. A number of additional mutants were included in this experiment when preliminary studies suggested that intragenic complementation occurred among the $A$ and $G$ mutants, and also that the $E$ and $G$ mutants, which did not accumulate Paulypositive imidazoles (Kloos \& Pattee, 1965) could be differentiated by their position within the histidine region. The results of this experiment did not show intragenic complementation within either the $C$ gene (mutants hisC: $-90,-14,-23$ were tested) or the $D$ gene (mutants hisD: $-36,-8,-42$, his AaD-63 were tested); but the significance of this result is limited by the numbers of mutants of each class examined. The $A$ gene was found to consist of three complementation units (his Aa: $-11,-31$, $-79,-83$, his A a D-63; his Ab: -1, -5, -6, -7, -10, -12, -17, -19, -20; his Ac-76) while the $G$ gene consisted of two complementation units (hisGa: $-40,-62,-74,-75 ;$ hisGb: -9 , $-13,-15,-38,-99)$. In addition, all genes $(E, A, B, C, D$ and $G)$ were capable of intergenic complementation with all other genes controlling the biosynthesis of histidine.

Table 4. Transduction analysis of the linkage relationships of the $E, A$ and B mutants of Staphylococcus aureus by the 'best-fit' method

Phage 83 prepared on each donor was used to infect $2 \cdot 5-3.5 \times 10^{10}$ cocci of each recipient at a multiplicity of $2 \cdot 0-3 \cdot 5$. The transduction frequency is the average number of prototrophic transductants recovered per $0.1 \mathrm{ml}$. of a $1 / 10$ dilution of the transduction suspension inoculated in duplicate on ES agar. The reversion frequency is the average number of prototrophic colonies recovered from uninfected coccal suspensions which were diluted and plated in the same manner as the transduction suspensions.

Transduction frequencies obtained using donor:

\begin{tabular}{|c|c|c|c|c|c|c|c|c|c|}
\hline Recipient & $\begin{array}{l}\text { Reversion } \\
\text { frequency }\end{array}$ & 655 & $\begin{array}{c}\text { hisE- } \\
49\end{array}$ & $\begin{array}{c}\text { his } A a- \\
31\end{array}$ & $\begin{array}{c}\text { hisAa- } \\
\quad 11\end{array}$ & $\begin{array}{c}h i s A b- \\
5\end{array}$ & $\begin{array}{c}\text { his } A b- \\
10\end{array}$ & $\begin{array}{c}\text { his Ac- } \\
76\end{array}$ & $\begin{array}{c}\text { hisB- } \\
2\end{array}$ \\
\hline hisE-49 & 7 & 118 & $\mathbf{0}$ & 10 & 20 & 35 & 21 & 28 & 36 \\
\hline his $A a-31$ & $\mathbf{3}$ & 132 & 42 & 0 & 20 & 39 & 34 & 32 & 68 \\
\hline his $A a-11$ & 10 & 140 & 44 & 17 & 9 & 41 & 30 & 38 & 60 \\
\hline his $A b-5$ & 4 & 119 & 72 & 64 & 60 & $\mathbf{3}$ & $\mathbf{2}$ & 10 & 26 \\
\hline$h i s A b-10$ & 10 & 136 & 65 & 51 & 52 & 11 & 6 & 16 & 23 \\
\hline his $A c-76$ & $\mathbf{0}$ & 141 & 66 & 62 & 60 & $\mathbf{2 6}$ & 6 & $\mathbf{0}$ & 10 \\
\hline hisB-2 & 14 & 332 & 331 & 288 & 295 & 242 & 152 & 36 & 16 \\
\hline
\end{tabular}

The order of $E, A$, and $B$ genes and the order of genes controlling histidine biosynthesis

To determine the order of complementation units within the $A$ gene and to verify the location of the $\boldsymbol{E}$ gene, a second transduction analysis was made by the 'bestfit' method. The results of this experiment (Table 4) established the order of genes and complementation units to be $E, A a, A b, A c, B$. The precise order of mutant sites within the complementation units could not be determined unambiguously from the data. As an example, mutants $h i s A b-5$ and $h i s A b-10$ could exist in an order other than that used to list the mutants in Table 4. 
Based upon the results of the 'best-fit' analyses, the ratio tests, and the complementation analysis, the histidine region of the chromosome of Staphylococcus aureus consists of the genetic arrangement depicted in Fig. 1. Included in Fig. 1 is the tentative pathway used by $S$. aureus for the biosynthesis of L-histidine as determined by the accumulation studies (Kloos \& Pattee, 1965).
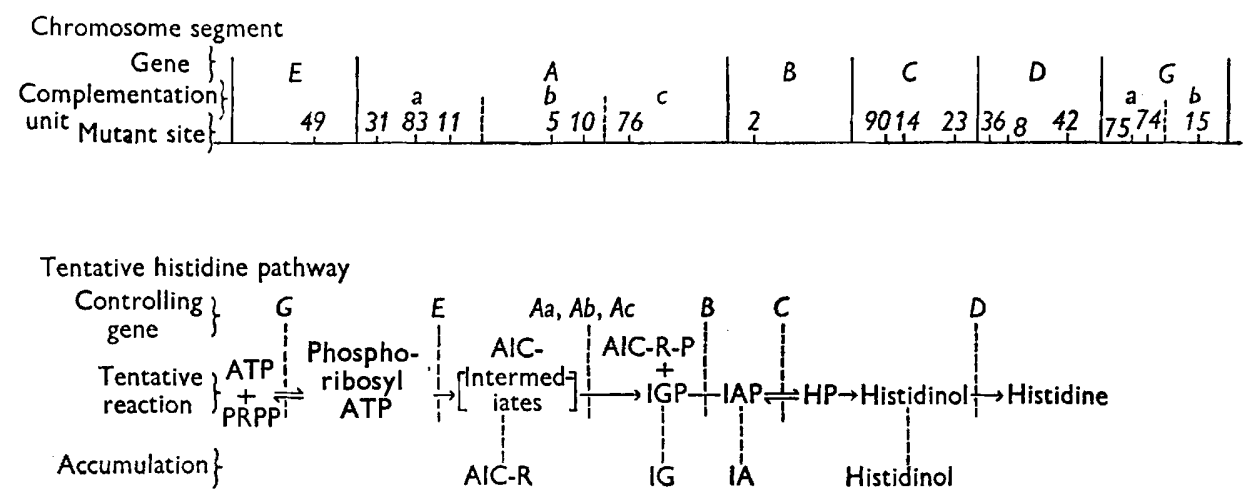

Fig. 1. A genetic map of the histidine region of the chromosome of Staphylococcus aureus, and the pathway used for the biosynthesis of histidine in this bacterium. Abbreviations used are: ATP $=$ adenosine triphosphate; PRPP $=$ phosphoribosylpyrophosphate; AIC = 5-amino-4-imidazolecarboxamide; AIC-R = 5-amino-1-ribosyl4-imidazolecarboxamide; AIC-R-P = 5-amino-1-(5'-phosphoribosyl)-4-imidazolecarboxamide; IGP = imidazoleglycerol phosphate; IG = imidazoleglycerol; IAP = imidazoleacetol phosphate; $\mathbf{I A}=$ imidazoleacetol; $\mathbf{H P}=$ histidinol phosphate.

\section{DISCUSSION}

In the previous paper (Kloos \& Pattee, 1965) evidence was presented that the biosynthesis of L-histidine in Staphylococcus aureus proceeds via a pathway which closely resembles the pathway found in Salmonella typhimurium. The results of the present study have shown that a corresponding similarity between the two species exists with respect to the genetic control of histidine biosynthesis. It is apparent that certain features of the histidine region of the chromosome of $S$. aureus require further study, particularly the $B$ and $E$ genes, each of which has been represented in the present work by only two mutants. None the less, sufficient information is available to permit certain comparisons to be made concerning the two species. The $A$ gene of $S$. aureus, defined by a study of accumulations (Kloos \& Pattee, 1965) consists of three complementation units as demonstrated by abortive transduction. It is most probable that this apparent intragenic complementation within the $\boldsymbol{A}$ gene is a reflexion of the fact that these complementation units actually are three different gene loci which correspond in function to one or more of the $E, A, H$ or $F$ genes of $S$. typhimurium. This possibility is supported by the close resemblance of the $A$ mutants of $S$. aureus to the $E, A, H$ and $F$ mutants of $S$. typhimurium with respect to their accumulations. Clarification of this question can be obtained by a study of the enzymes which are affected by mutations within the various complementation units of the $A$ gene of $S$. aureus. The demonstration of complementation within the $G$ gene of $S$. aureus, and the absence of a similar phenomenon in $\boldsymbol{S}$. typhimurium (Ames \& Hartman, 1963), represent a definite difference between the two species. This observation is of some interest in view of the demonstration by 
Martin (1963) that the enzyme produced under the control of the $G$ gene in $S$. typhimurium possesses a subunit structure.

One of the most striking features of the histidine region in Staphylococcus aureus is the correlation which exists between the position of a mutant site within the histidine region and the frequency with which that mutant is transduced to histidine independence by the parent strain (i.e. recipient capacity). This relationship is indicated by the data shown in Table 1 , which has been summarized according to mutant class in Table 5. When the mutants are arranged in an order of increasing recipient capacity, the order of mutant sites is in close agreement with the order determined by the ratio test and the 'best-fit' method of analysis. While there is some overlapping between the limits of recipient capacity of mutants of adjacent classes, the average recipient capacity for the mutant classes increases in a linear order which is identical with the order of gene loci. The only major discrepancy in this order is the overlapping which occurs between mutants of classes $C$ and $D$. This overlapping is explained by the sensitivity of $S$. aureus strain 655 and mutants of this strain to L-histidinol (Kloos \& Pattee, 1965), and the secretion of histidinol into the culture medium by the $D$ mutants, which may have caused a decrease in the observed transduction frequencies below the actual values.

Table 5. The relationship between the complementation unit or gene and the recipient capacity of histidine-dependent mutants of Staphylococcus aureus

\begin{abstract}
Recipient capacity is expressed in terms of the transduction frequencies obtained with phage $83 / 655$. Phage $83 / 655$ was used to infect $2 \cdot 5-3.5 \times 10^{10}$ cocci of each recipient at a multiplicity of 2.0 to 3.5 . The transduction frequency is the average number of prototrophic transductants recovered per $0.1 \mathrm{ml}$. of a $1 / 10$ dilution of the transduction suspension inoculated in duplicate on to ES agar.
\end{abstract}

\begin{tabular}{cccc}
$\begin{array}{c}\text { Comple- } \\
\text { mentation } \\
\text { unit or gene }\end{array}$ & $\begin{array}{c}\text { No. of } \\
\text { mutants } \\
\text { tested }\end{array}$ & & \multicolumn{2}{c}{ Recipient capacity } \\
$E$ & 2 & $100-118$ & Mean \\
$A a$ & 4 & $127-152$ & 109 \\
$A b$ & 9 & $113-151$ & 138 \\
$A c$ & 1 & 141 & 141 \\
$B$ & 2 & $215-332$ & 273 \\
$C$ & 8 & $322-518$ & 393 \\
$D$ & 29 & $334-677$ & 481 \\
$G a$ & 4 & $\mathbf{9 2 4 - 1 3 3 9}$ & 1192 \\
$G b$ & 5 & $\mathbf{9 9 0 - 1 3 8 9}$ & 1248
\end{tabular}

As a result of transduction studies in Salmonella typhimurium Ozeki (1959) suggested that the donor fragments which participate in transduction by phage PLT 22 are uniform and predetermined in size. While it is realized that other explanations exist, the theory of Ozeki (1959) appears best to explain the direct linear relationship which exists between the position of a mutant site within the histidine region and the recipient capacity of that mutant, and the inequalities obtained in reciprocal transduction between pairs of mutants. According to this hypothesis, and based on the data of the present study, the chromosome of Staphylococcus aureus always fragments immediately to the 'left' of the $E$ gene of the histidine region (Fig. 1) during formation of the donor fragments. The result would be that all, or nearly all, of the donor fragments which participate in transductions involving 
the histidine region would include the entire histidine region near this end of the fragment and in the same orientation. The donor fragment is assumed to extend beyond the 'right' end of the histidine region for a considerable distance, as evidenced by the high recipient capacity values obtained with the $G$ mutants. Therefore, the frequency of transduction of a mutant by the parent strain is determined by the position of that mutant with respect to the terminus of the donor fragment (Fig. 2). A mutant whose wild-type allele is located near the terminus of the donor fragment, such as mutant his Aa-83 (Fig. $\mathbf{2 A}$ ), permits crossing over to

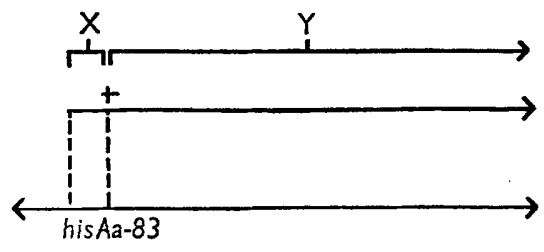

A

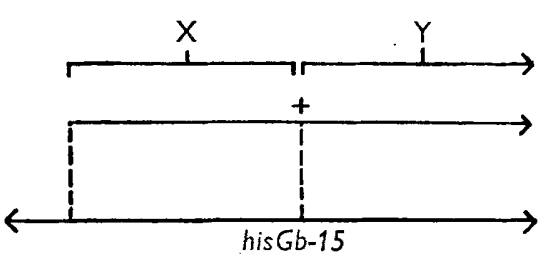

$B$

Fig. 2. Diagrammatic representation of the positions of the mutant sites in transductions of Staphylococcus aureus mutants his $A a-83$ and $h i s G b-15$ by the parent strain. The lower line represents the recipient chromosome and the upper line the donor fragment. The distances between the mutant sites and the terminal points of the donor fragment are designated by the letters $\mathbf{X}$ and $\mathbf{Y}$.
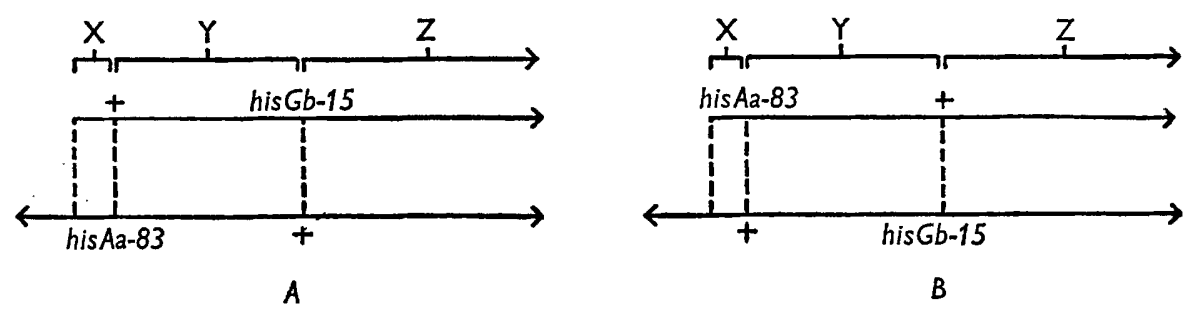

Fig. 3. Diagrammatic representation of the positions of the mutant sites in reciprocal transductions between Staphylococcus aureus mutants his Aa-83 and hisGb-15. The lower line represents the recipient chromosome and the upper line the donor fragment. The distances between the two mutant sites and the distances between the mutant sites and the terminal points of the donor fragment are designated by the letters $\mathbf{X}, \mathbf{Y}$ and $\mathrm{Z}$.

occur to the 'left' of the mutant site only within the small distance X. Mutants such as $h i s G b$ - 15 (Fig. $2 B$ ), whose wild-type allele is located at a greater distance from the terminus of the donor fragment, permit greater opportunity for cross-over to occur within distance $\mathbf{X}$. Distance $\mathbf{Y}$ in both instances is sufficiently great that no detectable restriction in cross-over occurs to the 'right' of the recipient mutant site. Fig. 3 illustrates the effect of this situation on the results of inter-mutant transductions, in which inequalities in the frequencies of recombination in reciprocal crosses are observed. When mutant his $\mathrm{Aa}-83$ is used as a recipient with mutant his $G b$-15 (Fig. $3 A$ ), one cross-over within distance $\mathrm{X}$ and another within distance $\mathrm{Y}$ are required. When mutant his $A a-83$ is used as the donor in the same genetic cross (Fig. $3 B$ ), one cross-over must occur within distance $Y$, and the other within distance $Z$. Thus, in crosses involving two mutants, the frequency with which prototrophic recombinants are obtained is restricted by distance $\mathrm{Y}$ in all cases, 
and by distance $\mathbf{X}$ separating the recipient mutant site from the terminus of the donor fragment when the recipient mutant site is closer to the terminus of the donor fragment than is the donor mutant site. This effect is reflected in the inequalities of reciprocal transduction frequencies, especially when these frequencies result from crosses between $A$ and $G$ mutants, where distance $\mathrm{Y}$ is maximal and distance $\mathrm{X}$ is minimal.

According to the above hypothesis, the incidence of abortive transduction obtained with all mutants after infection with phage propagated on the parent strain should be constant. While extensive data concerning this point has not been obtained, preliminary results confirm this prediction. In addition, the restriction in recombination due to the position of the histidine region within the donor fragment may affect the results of the ratio test. When the results of the ratio test are compared with the data of Hartman, Loper \& Serman (1960), the ratios obtained in the present study are significantly higher than those obtained in similar experiments in Salmonella typhimurium. As would be expected, this difference is most apparent in crosses in which $\boldsymbol{A}$ and $\boldsymbol{E}$ mutants are used as donors and $\boldsymbol{D}$ mutants are used as recipients.

This study was supported in part by Public Health Service Research Grant AI-04202 from the National Institute of Allergy and Infectious Diseases, and by Grant GB-2325 from the National Science Foundation.

\section{REFERENCES}

Ames, B. N. \& Hartman, P. E. (1962). Genes, enzymes, and control mechanisms in histidine biosynthesis. In The Molecular Basis of Neoplasia, pp. 322-45. Austin, Texas: University of Texas Press.

Ames, B. N. \& Hartman, P. E. (1963). The histidine operon. Cold Spr. Harb. Symp. quant. Biol. 28, 349.

Ephrati-Euzur, E., Srinivasan, P. R. \& Zamenhof, S. (1961). Genetic analysis, by means of transformation, of histidine linkage groups in Bacillus subtilis. Proc. natn. Acad. Sci., U.S.A. 47, 56.

HaAs, F., Mitchell, M. B., Ames, B. N. \& Mrtchell, H. K. (1952). A series of histidineless mutants of Neurospora crassa. Genetics, 37, 217.

Hartman, P. E. (1956). Linked loci in the control of consecutive steps in the primary pathway of histidine synthesis in Salmonella typhimurium. Genetic Studies with Bacteria, Publ. Carneg. Instn. no. 612, p. 35.

Hartman, P. E., Hartman, Z. \& Serman, D. (1960). Complementation mapping by abortive transduction of histidine requiring Salmonella mutants. J. gen. Microbiol. 22, 354 .

Hartman, P. E., Loper, J. C. \& Serman, D. (1960). Fine structure mapping by complete transduction between histidine requiring Salmonella mutants. J. gen. Microbiol. 22, 323.

Kloos, W. E. \& Pattee, P. A. (1965). A biochemical characterization of histidine-dependent mutants of Staphylococcus aureus. J. gen. Microbiol. 39, 185.

LOPER, J. C. (1960). Gene-enzyme relationships in histidine biosynthesis in Salmonella. Ph.D. Thesis. Baltimore, Maryland: The Johns Hopkins University.

LOPER, J. C. (1961). Enzyme complementation in mixed extracts of mutants from the Salmonella histidine B locus. Proc. natn. Acad. Sci., U.S.A. 47, 1440.

Martin, R. G. (1963). The first enzyme in histidine biosynthesis: the nature of feedback inhibition by histidine. J. biol. Chem. 238, 257.

OzeKI, H. (1959). Chromosome fragments participating in transduction in Salmonella typhimurium. Genetics, 44, 457. 
Pattee, P. A. \& Baldwin, J. N. (1961). Transduction of resistance to chlortetracycline and novobiocin in Staphylococcus aureus. J. Bact. 82, 875.

Pattee, P. A. \& Baldwin, J. N. (1962). Transduction of resistance to some macrolide antibiotics in Staphylococcus aureus. J. Bact. 84, 1049.

Sмiтh, D. W. E. \& Ames, B. N. (1964). Intermediates in the early steps of histidine biosynthesis. J. biol. Chem. 239, 1848.

Webber, B. \& CASE, M. (1960). Genetic and biochemical studies of histidine-requiring mutants of Neurospora crassa. I. Classification of mutants and characterizations of mutant groups. Genetics, 45, 1605. 